\title{
Phase Quadrature Backbone Curve for Nonlinear Modal Analysis of Nonconservative Systems
}

\author{
Martin Volvert and Gaëtan Kerschen \\ Department of Aerospace and Mechanical Engineering \\ University of Liège
}

\begin{abstract}
Nonlinear normal modes (NNMs) are often used to predict the backbone of resonance peaks in nonlinear frequency response functions. Regardless of the definition considered, one important limitation remains, i.e., the NNMs require a multi-point, multi-harmonic external forcing to be excited. To address this limitation, the present study proposes a new definition of NNMs, termed phase quadrature backbone curve (PQBC). The advantage of PQBC is that an actual solution of the nonlinear frequency response obtained under mono-point, mono-harmonic external forcing is followed for which phase quadrature of a selected degree of freedom is achieved. Additionally, super and subharmonic resonance peaks can be captured by adapting the phase quadrature condition. Finally, no post-processing is required to get amplitude-forcing relations of the NNMs. Isolated responses can, in turn, be predicted from these relations.
\end{abstract}

Keywords: Nonlinear vibrations, Harmonic forcing, Nonlinear modal analysis, Resonances.

\section{INTRODUCTION}

NNMs are used for modal analysis of nonlinear systems. Considering the unforced and undamped system, they can be defined as a (nonnecessarily synchronous) periodic motion [1]. To be excited, NNMs require a multi-point, multi-harmonic external forcing equal to the dissipative terms and in phase quadrature with each harmonic component of the displacement, as shown in [2].

In the presence of damping, the aforementioned definition can no longer be used. To address this issue, a new definition of NNMs accounting for linear and nonlinear damping was proposed by Krack [3], in which periodicity is enforced by a massproportional damping term, i.e., a fictitious force. Phase quadrature is obtained between each of the displacement harmonics and the fictitious force term. However, a multi-point, multi-harmonic forcing is still required for exciting the considered NNM, which is in contrast with the fact that nonlinear frequency responses are usually obtained under mono-point, mono-harmonic forcing.

In this study, a new NNM definition is proposed, which relies on the phase quadrature between a mono-point, mono-harmonic forcing and the first harmonic component of the displacement of the excited degree of freedom. This definition can also be extended to super and subharmonic resonance peaks by imposing phase quadrature between a higher or lower harmonic component of the displacement and of the external forcing.

\section{PHASE QUADRATURE BACKBONE CURVE}

Similar to what was achieved in [3], the basic idea of the PQBC definition is to introduce a fictitious external forcing to enforce periodicity. However, unlike [3], the fictitious forcing, which plays the role of mono-point and mono-harmonic forcing, only involves the first harmonic component of the velocity at the selected degree of freedom:

$$
M \ddot{x}+C \dot{x}+K x+f_{n l}(x, \dot{x})-\xi I_{l} \dot{x}_{1 h}=0
$$

where $1 h$ stands for first harmonic, $I_{l}$ is an $n \times n$ matrix filled with 0 s and one 1 on the $l$-th diagonal term, selecting the excited degree of freedom, and $\xi$ is the pseudo-amplitude of the fictitious forcing. In addition, super and subharmonics backbone curves 
can also be predicted by carefully selecting the harmonic component of the velocity in the fictitious forcing. Specifically, for super and subharmonic resonances, higher and lower than 1 harmonic components of the displacement are in phase quadrature with the forcing. Equation (3) thus becomes:

$$
M \ddot{x}+C \dot{x}+K x+f_{n l}(x, \dot{x})-\xi I_{l} \dot{x}_{n h}=0
$$

for the $n$-th superharmonic resonance, and:

$$
M \ddot{x}+C \dot{x}+K x+f_{n l}(x, \dot{x})-\xi I_{l} \dot{x}_{\frac{1}{n} h}=0
$$

for the $n$-th subharmonic resonances.

\section{NUMERICAL RESULTS}

The PQBC definition is first demonstrated using a simple Duffing oscillator:

$$
m \ddot{x}+c \dot{x}+k x+k_{n l} x^{3}=0
$$

The results are displayed in Figure 1. In addition to the fundamental resonance, the system presents super and subharmonic resonances, the loci of which are all accurately predicted using the PQBC NNMs. It should be noted that the subharmonic resonance only exists from a certain amplitude of the external forcing.

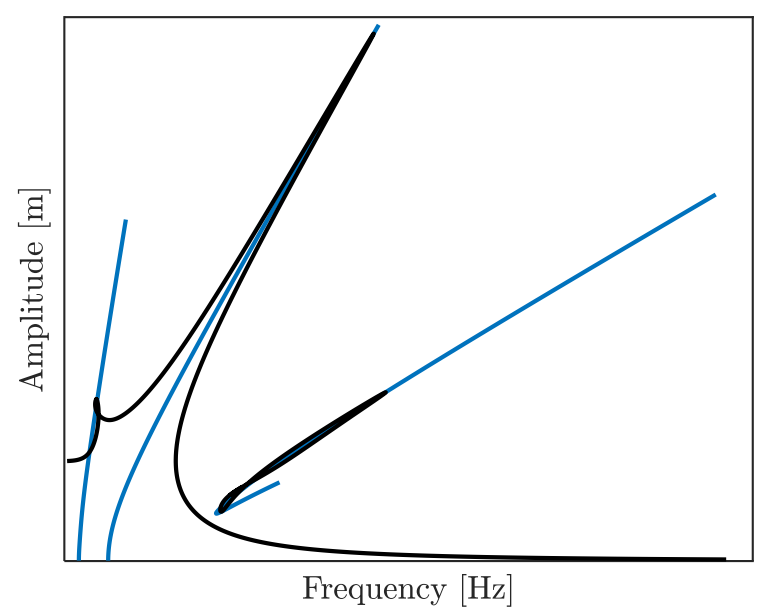

Figure 1: Nonlinear frequency response (in black) of the Duffing oscillator and PQBC NNMs (in blue).

Second, the method is applied to a two-degree-of-freedom system:

$$
\left\{\begin{array}{c}
m_{1} \ddot{x}_{1}+\left(c_{1}+c_{12}\right) \dot{x}_{1}-c_{12} \dot{x}_{2}+\left(k_{1}+k_{12}\right) x_{1}-k_{12} x_{2}+k_{n l} x_{1}^{3}=F \sin \omega t \\
m_{2} \ddot{x}_{2}+\left(c_{2}+c_{12}\right) \dot{x}_{2}-c_{12} \dot{x}_{1}+\left(k_{2}+k_{12}\right) x_{2}-k_{12} x_{1}=0
\end{array}\right.
$$

The undamped NNMs of this system feature several modal interactions. However, in practice, they can be annihilated by damping. Using PQBC, it is possible to accurately determine whether modal interactions are still present or not in the presence of damping. Specifically, the PQBC NNMs in Figure 2 clearly indicate that the modal interaction is eliminated by damping, which is confirmed by the calculation of the nonlinear frequency responses. 


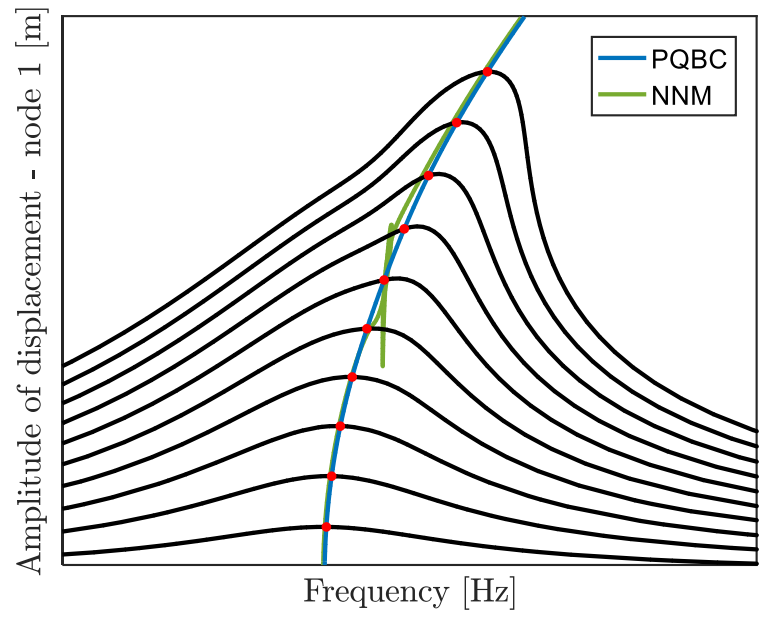

Figure 2: Comparison between undamped NNMs (green) and PQBC NNMs (blue). The red dots represent the phase quadrature points of the nonlinear frequency responses (black).

\section{CONCLUSION}

This study has introduced a new definition of NNMs, termed phase quadrature backbone curve (PQBC). $\mathrm{PQBC}$ is such that phase quadrature is enforced between the excited degree of freedom and the external forcing. It has been shown that PQBC can calculate accurately the loci of fundamental, super and subharmonic resonances.

\section{REFERENCES}

[1] G. Kerschen, M. Peeters, J.C. Golinval et A. Vakakis, «Nonlinear normal modes, Part I: A useful framework for the structural dynamicist,» Mechanical Systems and Signal Processing, vol. 23, pp. 170-194, 2009.

[2] M. Peeters, G. Kerschen et J.C. Golinval, «Dynamic testing of nonlinear vibrating structures using nonlinear normal modes,» Journal of Sound and Vibration, vol. 330, pp. 486-509, 2011.

[3] M. Krack, «Nonlinear modal analysis of nonconservative systems: Extension of the periodic motion concept,» Computers and Structures journal, vol. 154, pp. 59-71, 2015. 